\title{
Factors Affecting Product Supply in the Domestic Agrarian Market in Azerbaijan
}

\author{
Sugra Ingilab Humbatova ${ }^{1,2,3}$ \& Natig Gadim-Oglu Hajiyev ${ }^{3,4}$ \\ 1 Department of Economy and Management, International Center for Graduate Education, Azerbaijan State \\ University of Economics (UNEC), Baku, Azerbaijan \\ ${ }^{2}$ Department of World Economy, Baku Engineering University (BEU), Khirdalan, Absheron, Azerbaijan \\ ${ }^{3}$ Ministry of Agriculture of Azerbaijan, Agrarian Science and Innovation Center, Azerbaijan \\ ${ }^{4}$ Department of Regulation of Economy, Azerbaijan State University of Economics (UNEC), Baku, Azerbaijan \\ Correspondence: Natig Gadim-Oglu Hajiyev, Istiqlaliyyat Str. 6, Baku, AZ-1001, Azerbaijan.
}

Received: July 1, 2020

doi:10.5430/rwe.v11n5p246
Accepted: August 14, 2020

Online Published: September 3, 2020

URL: https://doi.org/10.5430/rwe.v11n5p246

\begin{abstract}
The purpose of the study was to study, analyze, evaluate and forecast the factors affecting the formation of product supply in the domestic agrarian market. Socio-economic aspects of the factors influencing the growth of product supply in agrarian market in the country were considered for the purpose of the research. Statistical and econometric analysis was carried out by the method of generalization, grouping, systematic approach, the role and place of factors influencing the growth of product supply in agrarian market. The methodology used is based on econometric analysis of time series. The first step involves the formation of an order of integration of variables included in the model and utilizing several unit root tests such as the Augmented Dickey-Fuller (ADF), Phillips-Perron (PP) and Kwiatkowski-Phillips-Schmidt-Shin (KPSS) tests. The cointegration approach is based on the ARDL model and the boundary test. The relative volatility of some indicators, which indirectly depends on oil prices, has led to some limitations in the study. The originality of the research is a complex statistical and econometric analysis of the factors influencing the increase of product supply in the domestic agricultural market, and the scientific novelty is the determination of the dependence of product supply in the agricultural sector on government support, including investment. The application of the article in the development of this section on the impact of agricultural supply on food security is of practical importance.
\end{abstract}

Keywords: agriculture, agrarian market, econometric analysis, product supply, government support

\section{Introduction}

The situation in the agricultural sector affects the socio-economic development of the whole economy. The specific role assigned to the agricultural sector is conditioned, on the one hand, by the production of food products as a basis for the reproduction of human life and labor, and, on the other hand, by the production of raw materials for other industries. In other words, in essence, the development of the agricultural sector determines the level of economic security of the country, including food security. Today, the development of the agrarian sector is one of the main priorities of the state's socio-economic and agrarian policy. When analyzing and solving the problem of development of the agrarian sector and the formation and increase of product supply in the agrarian market, it is necessary to study them comprehensively with the solution of the problems of food security, employment in agriculture, increase of incomes of those engaged in agrarian production.

\section{Literature Review}

In recent decades, the potential contribution of agriculture to economic growth has been the subject of great controversy among representatives of developing economies (Titus, 2009). Some scholars argue that economic growth depends on the development of the agricultural sector (Schultz, 2002). Proponents argue that investment in agriculture is accompanied by the creation of institutions and infrastructure in other sectors, which is the basis for national economic growth (Timmer, 1995; Timmer,2002; Gardner et al., 2001). Researchers have noted that growth in the agricultural sector can play a catalytic role through resource conditions for the transition to rural incomes and the industrial economy (Eicher and Staatz, 1990; Dowrick and Gemmell, 1991; Datt and Ravallion, 1998; Thirtle et 
al., 2003). The focus of many developing countries on industrial development before the agricultural sector has led to some contrasts in economic growth and excessive differences in income distribution. Others have shown that there is a positive link between agriculture and economic growth, and that agriculture has an impact on economic growth (Wang et al., 2010).

Many scientists have turned to econometrics in their research in this area. For example, an econometric analysis of the relationship between the agricultural, industrial, and service sectors to GDP growth (Mirza and Moyen, 2015) concludes that any change in agricultural sector strategy will affect the economy and the majority of the population (Alam, 2008). In addition, the relationship between the agricultural production, services and trade sectors in Poland and Romania was analyzed using the complex regression equation of the impact of agriculture on GDP in China and the three African Sahara (SSA) countries (Andzio-Bika et al., 2004). evaluated by econometric model (Subramaniam and Reed.2009).

Conducted an econometric analysis of the relationship between per capita income, agriculture and manufacturing sectors in India (Singapore, 2015), empirical analysis of Nigeria's contribution to economic growth in agriculture (Tolulope and Chinonso, 2013), Nigeria's contribution to economic growth in agricultural credit (Anthony, 2010), Inter-sectoral adjustment and economic growth in Saudi Arabia have been analyzed as a basis for a successful longterm development strategy (Abdulkarim et al., 2014).

\section{Methodology and Methods}

The data were obtained from the State Statistics Committee of Azerbaijan.

Table 1. Data and internet resource

\begin{tabular}{ccc}
\hline Melon products & $M P$ & www.stat.gov.az \\
Production of cereals and legumes & $P C L$ & www.stat.gov.az \\
Harvesting of autumn wheat & $H A W$ & www.stat.gov.az \\
Collection of potatoes & $C P$ & www.stat.gov.az \\
Harvesting of vegetables & $H V$ & www.stat.gov.az \\
Harvesting of fruits and berries & $H F B$ & www.stat.gov.az \\
Harvesting of grapes & $H G$ & www.stat.gov.az \\
Harvesting of sugar beet & $H S B$ & www.stat.gov.az \\
Meat production & MtP & www.stat.gov.az \\
Milk production & $M k P$ & www.stat.gov.az \\
Egg production & WP & www.stat.gov.az \\
Bovine cattle & $B C$ & www.stat.gov.az \\
Sheep and goats & $S G$ & www.stat.gov.az \\
Birds & $B$ & www.stat.gov.az \\
Planting area & $P A$ & www.stat.gov.az \\
Technique Park & $T P$ & www.stat.gov.az \\
Agricultural workers & $A W$ & www.stat.gov.az \\
Import of agricultural machinery for land & $I A M L$ & www.stat.gov.az \\
Import of equipment for the food industry & $I E P A P$ & www.stat.gov.az \\
\hline
\end{tabular}

\subsection{Methodology}

The supply of products in the agricultural market includes many agricultural products in all categories of production: production of cereals and legumes, total harvest of winter wheat, potatoes, vegetables, fruits and berries, grapes, sugar beets, meat, milk and eggs, including The main factors influencing the dynamics of the economy, including GDP and gross agricultural output by all categories of farms, are the number of cattle, sheep and goats, birds in all categories, agricultural machinery for cultivation, cultivation of agricultural crops. areas, agriculture, forestry and fisheries investment, the fleet of major types of agricultural machinery, the number of employees in agriculture, forestry and 
fisheries, the volume of imported equipment for processing of agricultural products and food industry.

$$
\begin{gathered}
Y_{t}=f\left(X_{t}\right) \\
\log _{e}\left(Y_{t}\right)=a_{0}+\sum \log _{e}\left(X_{t}\right) \\
L Y_{t}=a_{0}+\alpha_{1} L X_{t}+\varepsilon_{t}
\end{gathered}
$$

In this regard, to determine and assess the impact of the factors listed on each type of product offer, equations were constructed using the Eviews 9 package to reflect the relationship between them. It was used as statistical information in the Internet data of the State Statistics Committee reflecting the last 23 years (1995-2018).

All exponents have been converted to a natural (e-based) logarithm (ln).

\subsection{Model ARDL}

To test the reliability of our results, and as an additional sensitivity test, we will first perform a distributed lag autoregression (ARDL) analysis proposed by Pesaran and Shin (1999) for each factor. The ARDL method has several important advantages. Dependent and independent variables can have different lag lengths. Probably the biggest advantage of the ARDL approach is that it can be used for both "I (0)" variables and "I (1)" variables. Traditional cointegration processes require both variables to be "I (1)", and most standard regression processes require stationary. If any variable is defined as I (2) or higher, the ARDL method cannot be used. The existence of a single root was suggested by Dickey and Fuller (1979) and Kvyatkovsky et al. (1992). These tests allow us to determine if we can use ARDL analysis for all models.

After evaluating the correct number of variants based on Akayke's information criteria, we first perform tests to confirm heteroskedasticity (Breusch and Pagan, 1979). We then check for the absence of a consistent correlation based on the LM Test. Parameter stability tests (Page, 1954 and Barnard, 1959) are tested. After the models have been properly evaluated, we perform a boundary test developed by Pesaran and other authors (Pesaran et al., 2001). This is necessary to assess whether there is a long-term relationship between the variables. If the results of the boundary test reject the hypothesis of zero, then we estimate the long-term coefficients (Table 4 and 5).

Before considering quantitative analysis methods, several single root tests will be used to verify the existence of a single root. This is the first time that Levin et al. (Levin et al., 2002), as well as Im et al. (Im et al., 2003) (Table 1). Additional versions of the test proposed by Phillips and Perron (1988) and Dickey and Fuller (1979) will also be used (Table 2). All of these tests are performed against a zero hypothesis about the existence of a single root and are performed through an autoregression procedure based on the appropriate "lag" number. The number of lags is determined by the information criterion proposed by Schwartz (Schvarz, 1978) (Table 3) .

Based on the results of stationary tests, it can be concluded that the ARDL analysis can be used in full. The full results of single root tests are given in the table. We built models with the number of delays suggested by the Akayke criterion.

The mathematical expression of the model is as the following:

$$
\Delta Y_{t}=a_{y}+\sum_{i=1}^{n} \beta_{y i} \Delta Y_{t-i}+\sum_{j=0}^{n} \gamma_{y i} \Delta X_{t-J}+\theta_{y 1} Y_{t-1}+\theta_{y 2} X_{t-1}+\varepsilon_{y t}
$$

Then we transform by equalizing long-term coefficients to 0 in equation $1\left(a_{x}+\theta y_{t-1}+\theta_{y x} x_{t-1}=0\right)$, and we can solve it in terms of $y$ :

$$
y_{t}=-\frac{a_{x}}{\theta}-\frac{\theta_{y x}}{\theta} x_{t}+\varepsilon_{t}
$$

In this phase, long-term white noise error $\left(E C T_{t}\right)$ is calculated and inserted into the equation instead of long-term coefficients $\left(\theta y_{t-1}+\theta_{y x} x_{t-1}\right)$. Subsequently, assessment is done and the stability of cointegration relations is checked again. The mathematical function of evaluating model is as the following:

$$
\begin{gathered}
\Delta Y_{t}=a_{y}+\sum_{i=1}^{n} \beta_{y i} \Delta Y_{t-i}+\sum_{j=0}^{n} \gamma_{y i} \Delta X_{t-J}+\mu E C T_{t-1}+\varepsilon_{y t} \\
E C T_{t-1}=-\frac{a_{x}}{\theta}-\frac{\theta_{y x}}{\theta} x_{t}
\end{gathered}
$$


So, $y_{t}$ or $x_{t}$ is true value of dependent variable. $\left(-\frac{a_{x}}{\theta}-\frac{\theta_{y x}}{\theta} x_{t}\right)$ is calculated value according to long-term equation (equation 1). In equation 3 , if $\mu$ is between -1 and 0 and statistically important, then the cointegration realtions are constant. Deviation for short term period is inclined to be corrected towards long term relation. In case any serious calculation error is not noted, $\mu$ is getting close to $\theta$ coefficient in equation 1 , sometimes gets equal value.

At the first phase, regression analysis for non-original stationary but the same-level differentiated stationary (I(1)) variables is assessed. So, for the case of two variables:

$$
y_{t}=\alpha_{0}+\alpha_{1} x_{t}+\varepsilon_{t}
$$

Thus, $\alpha_{0}$ and $\alpha_{1}$ - regression coefficients, $y$ and $x$-dependent and independent variables, $\varepsilon$ - white noise error, $t$ - time. Having assessed regsression analysis, the next phase is to check whether $\varepsilon$ is white noise error. If $\varepsilon_{t}$ is stationary, there will be cointegration relations among these variables. Accordingly, it will be considered as long-term equations. At the last phase, ECM is assessed by using delayed white noise error $\left(\varepsilon_{t-1}\right)$ and converting cause-effect relations into stationary one.

$$
\Delta Y_{t}=a_{y}+\sum_{i=1}^{n} \beta_{y i} \Delta Y_{t-i}+\sum_{j=0}^{n} \gamma_{y i} \Delta X_{t-J}+\mu E C T_{t-1}+\varepsilon_{y t}
$$

Thus, $a_{y}, \beta_{y i}, \gamma_{y i}$ and $\mu$ coefficiants are mentioned. $n$ is a optimum delayed measure and $\varepsilon$ is a white noise error of the model. In case of having constant cointegration relations, Error Correction Term - ECT, thus $E C T_{t-1}$ coefficient $\mu$ is negative and statistically important. Usually, this changes -1 and 0 . If it is greater than -1 , this correction process is going to be high.

\section{Empirical Results}

\subsection{Results of Unit Root Tests}

As mentioned earlier, we begin by testing the integration of different variables using ADF, PP, and KPSS tests. The results of the three single root tests are given in Table 1 and Table 2. Approximately all three tests give the same results, confirming the reliability of our results. We can assume that none of the variables are integrated into the second level.

\begin{tabular}{|c|c|c|c|c|c|}
\hline Variable & $\begin{array}{l}\text { Levin, Lin and } \\
\text { Chu t }\end{array}$ & $\begin{array}{c}\text { Im, Pesaran and } \\
\text { Schin W-stat }\end{array}$ & $\begin{array}{l}\text { ADF-Fischer Chi } \\
\text { Square }\end{array}$ & $\begin{array}{c}\text { PP-Fischer Chi } \\
\text { Square }\end{array}$ & Conclusion \\
\hline$L M P$ & $-5.87618 * * *$ & $-4.03569 * * *$ & $69.6466^{* * *}$ & $27.2097^{*}$ & $\underline{\mathrm{I}(0)}$ \\
\hline$L P C L$ & $-5.51027 * * *$ & $-3.22822^{* * * *}$ & $62.4679 * * *$ & 19.4140 & $\underline{\mathrm{I}(1)}$ \\
\hline In first difference & $-11.2449^{* * *}$ & $-10.0091 * * *$ & $102.774 * * *$ & $114.758^{* * *}$ & \\
\hline LHAW & $-5.72631 * * *$ & $-3.64076 * * *$ & $65.5986 * * *$ & 21.3751 & $\underline{\mathrm{I}(1)}$ \\
\hline In first difference & $-11.0004 * * *$ & $-9.94732 * * *$ & $102.493 * * *$ & $121.128^{* * *}$ & \\
\hline$L C P$ & $-6.41613^{* * *}$ & $-4.59198 * * *$ & $75.9687^{* * *}$ & $32.1249 * *$ & $\underline{\mathrm{I}(0)}$ \\
\hline$L H V$ & $-5.48198 * * *$ & $-3.59204 * * *$ & $65.1545^{* * *}$ & $24.4587^{*}$ & $\underline{\mathrm{I}(0)}$ \\
\hline LHFB & $-5.26788 * * *$ & $-3.10996 * * *$ & $61.9616^{* * *}$ & 17.8688 & $\underline{\mathrm{I}(1)}$ \\
\hline In first difference & $-12.3660 * * *$ & $-10.9360 * * *$ & $112.796 * * *$ & $131.956^{* * *}$ & \\
\hline$L H G$ & $-5.50569 * * *$ & $-3.70833 * * *$ & $66.2244 * * *$ & 20.2523 & $\underline{\mathrm{I}(1)}$ \\
\hline In first difference & $-10.5260 * * *$ & $-9.29163 * * *$ & $94.9867 * * *$ & $101.885^{* * *}$ & \\
\hline$L H S B$ & $-5.42467 * * *$ & $-3.30749 * * *$ & $62.9427 * * *$ & 19.0989 & $\underline{\mathrm{I}(1)}$ \\
\hline In first difference & $-10.7351 * * *$ & $-9.90403 * * *$ & $101.607 * * *$ & $108.801 * * *$ & \\
\hline$L M t P$ & $-5.67425 * * *$ & $-4.35735^{* * *}$ & $34.0310^{* * *}$ & $77.3266^{* * *}$ & $\underline{\mathrm{I}(0)}$ \\
\hline$L M k P$ & $-2.43421 * *$ & -1.28490 & $9.83982^{*}$ & $19.3031 * * *$ & $\underline{\mathrm{I}(0)}$ \\
\hline$L W P$ & $-3.84042 * * *$ & $-2.01582^{*}$ & $15.4843^{* *}$ & $15.4009^{* *}$ & $\underline{\mathrm{I}(0)}$ \\
\hline
\end{tabular}

Table 1. Unit root tests Panel

Note: values in the parenthesis represent the p value. $*$ and $* *$ indicate statistical significance at the respected 0.05 and 0.01 levels of significance. 
Table 2. Unit Root Tests (ADF, PP, KPSS)

\begin{tabular}{|c|c|c|c|c|c|c|c|c|}
\hline & & \multicolumn{2}{|c|}{ ADF } & \multicolumn{2}{|c|}{ Phillips-Perron } & \multicolumn{2}{|c|}{ KPSS } & \\
\hline & & Level & 1st difference & Level & 1st difference & Level & 1st difference & \\
\hline \multirow[t]{3}{*}{$L M P$} & Intercept & $-3.672634 * *$ & $-2.826665^{*}$ & $-3.986381 * * *$ & $-2.779897 *$ & $0.525222 * *$ & $0.494147 * *$ & $\mathrm{I}(0)$ \\
\hline & Trend \& Intercept & -1.661652 & $-3.739991 * *$ & -1.741905 & $-3.655090 * *$ & $0.167639 * *$ & 0.084111 & \\
\hline & None & 1.713418 & $-2.668529 * *$ & 1.263599 & $-2.591958 * *$ & & & \\
\hline \multirow[t]{3}{*}{$L P C L$} & Intercept & -1.669227 & $-5.173644 * * *$ & -1.983024 & $-6.238471 * * *$ & $0.632894 * *$ & $0.500000 * *$ & $\mathrm{I}(1)$ \\
\hline & Trend \& Intercept & -2.367686 & $-4.721812 * * *$ & -2.393640 & $-10.23087 * * *$ & $0.163990^{* *}$ & $0.500000^{* * *}$ & \\
\hline & None & 1.492375 & $-4.763710^{* * *}$ & 3.513391 & $-4.786334 * * *$ & & & \\
\hline \multirow[t]{3}{*}{$L H A W$} & Intercept & $-2.692781^{*}$ & $-4.537878 * * *$ & -2.589663 & $-7.683177 * * *$ & $0.579224 * *$ & $0.500000 * *$ & $\mathrm{I}(0)$ \\
\hline & Trend \& Intercept & -3.073752 & $-4.413949 * *$ & -2.942564 & $-8.095731 * * *$ & $0.126401^{*}$ & $0.500000^{* *}$ & \\
\hline & None & 0.695636 & $-4.568118 * * *$ & 2.087992 & $-5.278137 * * *$ & & & \\
\hline \multirow[t]{3}{*}{$L C P$} & Intercept & $-5.052829 * * *$ & -1.583892 & $-5.052829 * * *$ & $-3.200009^{* *}$ & $0.510241 * *$ & $0.564407 * *$ & $\mathrm{I}(0)$ \\
\hline & Trend \& Intercept & -2.065379 & $-4.754600 * * *$ & -2.261149 & $-4.752371 * * *$ & $0.184279^{* *}$ & $0.145119^{*}$ & \\
\hline & None & $-0.240001 * *$ & $-2.118538 * *$ & 1.499286 & $-3.030740 * * *$ & & & \\
\hline \multirow[t]{3}{*}{$L H V$} & Intercept & $-4.142045^{* * *}$ & $-5.271260 * * *$ & $-3.363969 * *$ & $-5.142171 * * *$ & $0.603988^{* *}$ & $0.367830 *$ & $\mathrm{I}(0)$ \\
\hline & Trend \& Intercept & -3.223991 & $-3.950730 * *$ & -2.135698 & $-5.178652 * * *$ & $0.167128^{* *}$ & $0.134206^{*}$ & \\
\hline & None & 1.948755 & $-1.893647^{*}$ & 2.570608 & $-4.458042 * * *$ & & & \\
\hline \multirow[t]{3}{*}{$L H F B$} & Intercept & -1.412475 & $-7.426656^{* * *}$ & -1.256485 & $-9.993472 * * *$ & $0.698321 * *$ & 0.251701 & $\mathrm{I}(0)$ \\
\hline & Trend \& Intercept & $-3.510947^{*}$ & $-7.446849 * * *$ & $-3.510947^{*}$ & $-17.50699 * * *$ & $0.156314 * *$ & $0.402438 * *$ & \\
\hline & None & 3.010484 & $-5.657066^{* * *}$ & 4.423357 & $-5.591175^{* * *}$ & & & \\
\hline \multirow[t]{3}{*}{$L H G$} & Intercept & -2.259763 & $-3.429420 * *$ & -2.261873 & $-3.356813^{* *}$ & 0.160782 & $0.462268^{*}$ & $\mathrm{I}(0)$ \\
\hline & Trend \& Intercept & $-4.785879 * * *$ & $-4.316617 * *$ & -2.960291 & $-4.377748 * *$ & $0.148096^{* *}$ & $0.171902 * *$ & \\
\hline & None & -0.808248 & $-3.496559 * * *$ & -0.694555 & $-3.425024 * * *$ & & & \\
\hline \multirow[t]{3}{*}{$L H S B$} & Intercept & -1.865900 & $-4.918137 * * *$ & -1.865900 & $-4.915771 * * *$ & $0.540506^{* *}$ & 0.101148 & $\mathrm{I}(1)$ \\
\hline & Trend \& Intercept & -2.203451 & $-4.826083 * * *$ & -2.253532 & $-4.824879 * * *$ & $0.128110^{*}$ & 0.048218 & \\
\hline & None & 0.655944 & $-4.886455^{* * *}$ & 0.710225 & $-4.886455^{* * *}$ & & & \\
\hline \multirow[t]{3}{*}{$L M t P$} & Intercept & $-3.153891 * *$ & $-3.179911 * *$ & $-8.982006^{* * *}$ & $-3.043430^{* *}$ & $0.706960 * *$ & $0.505161 * *$ & $\mathrm{I}(0)$ \\
\hline & Trend \& Intercept & -1.790276 & $-4.175078 * *$ & -1.938488 & $-4.287385^{* *}$ & $0.179437 * *$ & $0.191809^{* *}$ & \\
\hline & None & 8.096754 & $-1.696549 *$ & 6.549402 & -1.286720 & & & \\
\hline \multirow[t]{3}{*}{$L M k P$} & Intercept & -0.933688 & $-3.797633 * * *$ & -0.933688 & $-3.797633 * * *$ & $0.706708^{* *}$ & 0.144888 & $\mathrm{I}(0)$ \\
\hline & Trend \& Intercept & $-3.508246^{*}$ & $-4.032167 * *$ & -1.522557 & $-4.032167 * *$ & 0.064590 & 0.093693 & \\
\hline & None & 12.84266 & -1.152463 & 12.84266 & -0.648381 & & & \\
\hline \multirow[t]{3}{*}{$L W P$} & Intercept & -0.579388 & $-4.512587 * * *$ & -0.400480 & $-5.945121 * * *$ & $0.694680 * *$ & 0.267084 & $\mathrm{I}(0)$ \\
\hline & Trend \& Intercept & $-3.770215^{* *}$ & $-4.341819^{* *}$ & -2.626947 & $-5.713731 * * *$ & 0.102925 & $0.265775^{* * * *}$ & \\
\hline & None & 2.727636 & -3.496253 & 5.902792 & $-3.469321 * * *$ & & & \\
\hline \multirow[t]{3}{*}{$L B C$} & Intercept & $-3.787048 * * *$ & 3.163740 & $-5.826452 * * *$ & -0.276897 & $0.674576^{* *}$ & $0.661100 * *$ & $\mathrm{I}(0)$ \\
\hline & Trend \& Intercept & 1.904157 & $-5.809286^{* * *}$ & 5.049311 & $-5.642330 * * *$ & $0.193263^{* *}$ & $0.131984^{*}$ & \\
\hline & None & $-3.336230 * * *$ & -1.165348 & 3.680886 & -1.084073 & & & \\
\hline \multirow[t]{3}{*}{$L S G$} & Intercept & $-4.676262 * * *$ & -0.637542 & $-4.173382 * * *$ & -0.704705 & $0.643082 * *$ & $0.593403 * *$ & $\mathrm{I}(0)$ \\
\hline & Trend \& Intercept & -0.432821 & $-3.366218^{*}$ & 0.998314 & $-4.685849 * * *$ & $0.190570 * *$ & $0.127772 *$ & \\
\hline & None & 0.060277 & -0.862921 & 2.843871 & -0.820877 & & & \\
\hline \multirow[t]{3}{*}{$L B$} & Intercept & $-5.052829 * * *$ & -1.583892 & $-5.052829 * * *$ & $-3.200009 * *$ & $0.510241 * *$ & $0.564407 * *$ & $\mathrm{I}(0)$ \\
\hline & Trend \& Intercept & -2.065379 & $-4.754600 * * *$ & -2.261149 & $-4.752371 * * *$ & $0.184279 * *$ & $0.145119 * *$ & \\
\hline & None & -0.240001 & $-2.118538^{* *}$ & 1.499286 & $-3.030740 * * *$ & & & \\
\hline \multirow[t]{3}{*}{$L P A$} & Intercept & -0.469814 & $-4.247930 * * *$ & -0.559355 & $-4.234967 * * *$ & $0.605123^{* *}$ & 0.152014 & $\mathrm{I}(1)$ \\
\hline & Trend \& Intercept & -2.795675 & $-4.341401 * *$ & -2.699713 & $-4.391597 * *$ & 0.078160 & 0.097288 & \\
\hline & None & 0.962586 & $-4.175466^{* * *}$ & 1.085959 & $-4.160613^{* *}$ & & & \\
\hline$L T P$ & Intercept & -2.111025 & $-3.930289 * * *$ & -2.138881 & $-3.930868 * * *$ & $0.473754 * *$ & 0.185176 & $\mathrm{I}(1)$ \\
\hline & Trend \& Intercept & -1.726842 & $-4.107091 * * *$ & -1.862931 & $-4.057927 * *$ & $0.141264 *$ & 0.063890 & \\
\hline & None & -0.321736 & $-4.039617 * * *$ & -0.328185 & $-4.040257 * * *$ & & & \\
\hline$L A W$ & Intercept & 0.792477 & $-6.918204 * * *$ & 0.792477 & $-6.918204 * * *$ & $0.669293 * *$ & $0.479422 * *$ & $\mathrm{I}(1)$ \\
\hline & Trend \& Intercept & -3.128392 & $-7.724645^{* * *}$ & -3.168316 & $-9.519979 * * *$ & $0.171378 * *$ & 0.090568 & \\
\hline & None & 2.060966 & $-5.130067 * * *$ & 2.457790 & $-5.086439 * * *$ & & & \\
\hline LIAML & Intercept & -2.190783 & $-5.568428 * * *$ & $-2.917890^{*}$ & $-5.667217 * * *$ & $0.679513^{* *}$ & $0.500000 * *$ & $\mathrm{I}(0)$ \\
\hline
\end{tabular}




\begin{tabular}{|c|c|c|c|c|c|c|c|c|}
\hline & Trend \& Intercept & $-3.986444 * *$ & $-6.839234 * * *$ & -3.236761 & $-10.16664 * * *$ & $0.144527 *$ & $0.500000 * * *$ & \\
\hline & None & 0.915576 & $-4.254482 * * *$ & 1.405578 & $-4.714391 * * *$ & & & \\
\hline \multirow[t]{3}{*}{ LIEPAP } & Intercept & $-11.12009 * * *$ & $-4.638987 * * *$ & $-2.715192^{*}$ & $-4.638987 * * *$ & $0.574978^{* *}$ & 0.282622 & $\mathrm{I}(0)$ \\
\hline & Trend \& Intercept & $-6.278620 * * *$ & $-5.656698 * * *$ & -1.464107 & $-6.489772 * * *$ & $0.171095^{* *}$ & $0.140342 *$ & \\
\hline & None & 0.987038 & $-4.273847 * * *$ & 0.987038 & $-4.272079 * * *$ & & & \\
\hline \multirow[t]{3}{*}{ LIEFI } & Intercept & -2.287770 & $-3.837543 * * *$ & -2.214773 & $-5.691819 * * *$ & $0.454186^{*}$ & 0.256797 & $\mathrm{I}(1)$ \\
\hline & Trend \& Intercept & -2.490288 & $-4.026699 * *$ & -2.509444 & $-7.318474 * * *$ & $0.156570^{* *}$ & $0.180017 * *$ & \\
\hline & None & 0.389327 & $-5.261758 * * *$ & 0.961876 & $-5.631332 * * *$ & & & \\
\hline
\end{tabular}

Note: values in the parenthesis represent the $\mathrm{p}$ value. $*, * *$ and $* * *$ indicate statistical significance at the respected $0.1,0.05$, and 0.01 levels of significance.

\subsection{VAR Lag Order Selection Criteria}

In order to determine optimal lag for ARDL model, VAR Lag Order Selection Criteria was employed and we got the below-mentioned results (Table 3 ).

According to Table 3, optimum lag period for all models is $1(\mathrm{lag}=1)$ based on 2 accepted information criteria (AIC and $\mathrm{SC})$.

Table 3. VAR Lag order selection criteria

\begin{tabular}{cccccccc}
\hline & Lag & LogL & LR & FPE & AIC & SC & HQ \\
\hline$L M P$ & 1 & 98.70357 & $176.4856^{*}$ & $7.11 \mathrm{e}-11^{*}$ & $-3.713354^{*}$ & $-0.948672^{*}$ & $-3.018044^{*}$ \\
$L P C L$ & 1 & 90.67892 & $148.2528^{*}$ & $1.43 \mathrm{e}-10^{*}$ & $-3.015558^{*}$ & $-0.250877^{*}$ & $-2.320249^{*}$ \\
$L H A W$ & 1 & 94.05860 & $150.2221^{*}$ & $1.07 \mathrm{e}-10^{*}$ & $-3.309443^{*}$ & $-0.544762^{*}$ & $-2.614134^{*}$ \\
$L C P$ & 1 & 98.01940 & $155.7278^{*}$ & $7.55 \mathrm{e}-11^{*}$ & $-3.653861^{*}$ & $-0.889179^{*}$ & $-2.958551^{*}$ \\
$L H V$ & 1 & 98.59811 & $145.1637^{*}$ & $7.18 \mathrm{e}-11^{*}$ & $-3.704183^{*}$ & $-0.939502^{*}$ & $-3.008874^{*}$ \\
$L H F B$ & 1 & 112.7470 & $154.8050^{*}$ & $2.10 \mathrm{e}-11^{*}$ & $-4.934518^{*}$ & $-2.169836^{*}$ & $-4.239208^{*}$ \\
$L H G$ & 1 & 81.87888 & $159.1856^{*}$ & $3.07 \mathrm{e}-10^{*}$ & $-2.250337^{*}$ & $0.514344^{*}$ & $-1.555028^{*}$ \\
$L H S B$ & 1 & 64.46104 & $140.0340^{*}$ & $1.40 \mathrm{e}-09^{*}$ & $-0.735743^{*}$ & $2.028939^{*}$ & $-0.040433^{*}$ \\
$L M t P$ & 1 & 253.9867 & $201.7985^{*}$ & $1.76 \mathrm{e}-14^{*}$ & $-20.34667^{*}$ & $-19.35929^{*}$ & $-20.09835^{*}$ \\
$L M k P$ & 1 & 148.8730 & $191.8811^{*}$ & $7.87 \mathrm{e}-09^{*}$ & $-12.98845^{*}$ & $-12.69090^{*}$ & $-12.91836^{*}$ \\
$L W P$ & 1 & 45.34422 & $107.6644^{*}$ & $9.62 \mathrm{e}-05^{*}$ & $-3.576748^{*}$ & $-3.279191^{*}$ & $-3.506652^{*}$ \\
\hline
\end{tabular}

* indicates lag order selected by the criterion

LR: sequential modified LR test statistic (each test at $5 \%$ level)

FPE: Final prediction error

AIC: Akaike information criterion

SC: Schwarz information criterion

HQ: Hannan-Quinn information criterion

Table 4. Bounds test

\begin{tabular}{ccc}
\hline & ARDL Bounds Test & $\begin{array}{c}\text { Null Hypothesis of No } \\
\text { Long-Run Relationship }\end{array}$ \\
\hline ARDL $(1,1,1,0,1,1,1)$ C @ TREND & & Failed to reject \\
Rejected \\
ARDL $(1,0,0,1,0)^{2}$ & 3.448147 & Failed to reject \\
ARDL $(1,0,0,0,0,0,0)^{3}$ & $4.111522^{*}$ & Rejected \\
ARDL $(1,0,1,0,0,0,0)^{3}$ & 2.463027 & Failed to reject \\
ARDL $(1,1,1,1,1,1)^{4}$ & $4.540147^{* * *}$ & Failed to reject \\
ARDL $(1,1,0,1)^{6}$ & 2.198584 & Failed to reject \\
ARDL $(1,1,0,1,0,1,1)^{2}$ & 3.689006 & Failed to reject \\
ARDL $(1,0,0,0,1,0)^{5}$ & 3.466548 & Failed to reject \\
ARDL $(1,0,1,1)^{6}$ & 2.289684 & Failed to reject
\end{tabular}


$\operatorname{ARDL}(1,0,0)^{8}$

0.801822

Failed to reject

\begin{tabular}{ccccccccc}
\hline \multicolumn{1}{c}{ I0 Bound } \\
\hline & $10 \%$ & $5 \%$ & $2.5 \%$ & $1 \%$ & $10 \%$ & $5 \%$ & $2.5 \%$ & $1 \%$ \\
\hline 1 & 2.53 & 2.87 & 3.19 & 3.6 & 3.59 & 4 & 4.38 & 4.9 \\
2 & 3.03 & 3.47 & 3.89 & 4.4 & 4.06 & 4.57 & 5.07 & 5.72 \\
3 & 2.12 & 2.45 & 2.75 & 3.15 & 3.23 & 3.61 & 3.99 & 4.43 \\
4 & 2.75 & 3.12 & 3.49 & 3.93 & 3.79 & 4.25 & 4.67 & 5.23 \\
5 & 3.17 & 3.79 & 4.41 & 5.15 & 4.14 & 4.85 & 5.52 & 6.36 \\
6 & 2.72 & 3.23 & 3.69 & 4.29 & 3.77 & 4.35 & 4.89 & 5.61 \\
7 & 3.17 & 3.79 & 4.41 & 5.15 & 4.14 & 4.85 & 5.52 & 6.36 \\
8 & 4.04 & 4.94 & 5.77 & 6.84 & 4.78 & 5.73 & 6.68 & 7.84 \\
\hline
\end{tabular}

Table 4 reveals the cointegration relations among variables. Thus, there are the no-cointegration relation. In other words, there is a no long term relations. Thus, based on the Narayan (2005) table, F-statistics is below 5\% minimum indicator.

\subsection{ARDL-Results for Long Run Model}

Table 5. Long run coefficients

\begin{tabular}{|c|c|c|c|c|c|}
\hline & Variable & Coefficient & Std. Error & $\mathrm{t}-$ Statistic & Prob. \\
\hline \multirow[t]{9}{*}{$L M P$} & $L P A$ & 10.511971 & 46.802581 & 0.224602 & 0.8273 \\
\hline & $L T P$ & 2.374136 & 11.993740 & 0.197948 & 0.8475 \\
\hline & $L A W$ & -364.603883 & 1583.352396 & -0.230273 & 0.8230 \\
\hline & LIAML & -3.654995 & 16.282656 & -0.224472 & 0.8274 \\
\hline & LIEPAP & 1.431174 & 5.333134 & 0.268355 & 0.7945 \\
\hline & LIEFI & -1.741587 & 7.820332 & -0.222700 & 0.8287 \\
\hline & $\mathrm{C}$ & 2587.297687 & 11207.430292 & 0.230856 & 0.8226 \\
\hline & @TREND & 3.692734 & 16.106312 & 0.229272 & 0.8238 \\
\hline & \multicolumn{5}{|c|}{$\begin{array}{c}\text { Cointeq }=L M P-\left(10.5120 * L P A+2.3741 * L T P-364.6039 * L A W-3.6550 * \text { LIAML }+1.4312 * \text { LIEPAP }-1.7416^{*}\right. \\
\text { LIEFI }+2587.2977+3.6927 * @ \mathrm{TREND})\end{array}$} \\
\hline \multirow[t]{7}{*}{$L P C L$} & $L P A$ & 0.388436 & 0.168957 & 2.299020 & 0.0363 \\
\hline & $L T P$ & 0.080023 & 0.084367 & 0.948510 & 0.3579 \\
\hline & $L A W$ & -11.849017 & 1.986854 & -5.963708 & 0.0000 \\
\hline & LIAML & -0.014553 & 0.024280 & -0.599386 & 0.5579 \\
\hline & $\mathrm{C}$ & 90.101063 & 14.186338 & 6.351256 & 0.0000 \\
\hline & @TREND & 0.124099 & 0.020810 & 5.963394 & 0.0000 \\
\hline & \multicolumn{5}{|c|}{$\begin{array}{c}\text { Cointeq }=L P C L-\left(0.3884^{*} L P A+0.0800^{*} L T P-11.8490^{*} L A W-0.0146^{*} \text { LNSERIES } 18+90.1011+\right. \\
0.1241 * @ \text { TREND })\end{array}$} \\
\hline \multirow[t]{8}{*}{$L H A W$} & $L P A$ & 1.212078 & 0.919004 & 1.318904 & 0.2070 \\
\hline & $L T P$ & 0.309810 & 0.269822 & 1.148201 & 0.2689 \\
\hline & $L A W$ & -4.537886 & 4.027680 & -1.126675 & 0.2776 \\
\hline & LIAML & 0.029534 & 0.062751 & 0.470662 & 0.6447 \\
\hline & LIEPAP & 0.023965 & 0.054351 & 0.440941 & 0.6655 \\
\hline & LIEFI & 0.019393 & 0.077990 & 0.248664 & 0.8070 \\
\hline & $\mathrm{C}$ & 27.274317 & 22.517125 & 1.211270 & 0.2445 \\
\hline & $\begin{array}{r}\text { Cointeq }=\text { LHAV } \\
+0.0194 * L I E F I+27 .\end{array}$ & $A+0.3098^{*} L$ & $5379 * L A W+$ & 5* LIAML + & * LIEPAP \\
\hline \multirow[t]{5}{*}{$L C P$} & $L P A$ & -0.248363 & 0.400158 & -0.620663 & 0.5448 \\
\hline & $L T P$ & -0.515361 & 0.215746 & -2.388734 & 0.0315 \\
\hline & $L A W$ & -2.518426 & 1.906648 & -1.320866 & 0.2077 \\
\hline & LIAML & 0.004772 & 0.055957 & 0.085286 & 0.9332 \\
\hline & LIEPAP & 0.123184 & 0.044069 & 2.795251 & 0.0143 \\
\hline
\end{tabular}




\begin{tabular}{|c|c|c|c|c|c|}
\hline & LIEFI & 0.103593 & 0.061826 & 1.675541 & 0.1160 \\
\hline & $\mathrm{C}$ & 30.442406 & 12.924803 & 2.355348 & 0.0336 \\
\hline & \multicolumn{5}{|c|}{$\begin{array}{c}\text { Cointeq }=L C P-(-0.2484 * L P A-0.5154 * L T P-2.5184 * L A W+0.0048 * L I A M L+0.1232 * \text { LNSERIES } 19+ \\
0.1036 * \text { LIEFI + 30.4424 })\end{array}$} \\
\hline \multirow[t]{7}{*}{$L H V$} & $L P A$ & 0.251743 & 0.252946 & 0.995243 & 0.3431 \\
\hline & $L T P$ & -0.145584 & 0.130447 & -1.116042 & 0.2905 \\
\hline & $L A W$ & 5.399517 & 6.757426 & 0.799049 & 0.4428 \\
\hline & LIAML & 0.003915 & 0.037168 & 0.105339 & 0.9182 \\
\hline & LIEPAP & 0.153148 & 0.058781 & 2.605401 & 0.0262 \\
\hline & $\mathrm{C}$ & -33.887775 & 49.369653 & -0.686409 & 0.5081 \\
\hline & @ TREND & -0.053972 & 0.066643 & -0.809863 & 0.4369 \\
\hline \multirow[t]{5}{*}{ LHFB } & \multicolumn{5}{|c|}{$\begin{array}{c}\text { Cointeq }=\text { LHV }-\left(0.2517 * L P A-0.1456 * L T P+5.3995^{*} L A W+0.0039 * L I A M L+0.1531 * L I E P A P-33.8878-\right. \\
0.0540 * @ \text { TREND })\end{array}$} \\
\hline & LIAML & 0.136151 & 0.027642 & 4.925488 & 0.0002 \\
\hline & LIEPAP & 0.004019 & 0.033062 & 0.121551 & 0.9048 \\
\hline & LIEFI & 0.034757 & 0.046924 & 0.740706 & 0.4696 \\
\hline & $\mathrm{C}$ & 4.941408 & 0.312095 & 15.833031 & 0.0000 \\
\hline \multicolumn{6}{|c|}{ Cointeq $=$ LHFB $-(0.1362 *$ LIAML $+0.0040 *$ LIEPAP $+0.0348 *$ LIEFI +4.9414$)$} \\
\hline \multirow[t]{10}{*}{$L H G$} & $L P A$ & 0.738864 & 0.669883 & 1.102974 & 0.2959 \\
\hline & $L T P$ & -0.302245 & 0.306486 & -0.986163 & 0.3473 \\
\hline & $L A W$ & 42.668682 & 14.550896 & 2.932375 & 0.0150 \\
\hline & LIAML & 0.051365 & 0.081657 & 0.629040 & 0.5434 \\
\hline & LIEPAP & 0.237441 & 0.158081 & 1.502021 & 0.1640 \\
\hline & LIEFI & 0.087750 & 0.110463 & 0.794382 & 0.4454 \\
\hline & $\mathrm{C}$ & -311.024777 & 105.659333 & -2.943656 & 0.0147 \\
\hline & @TREND & -0.374579 & 0.150689 & -2.485774 & 0.0322 \\
\hline & \multicolumn{5}{|c|}{ Cointeq $=L H G-\left(0.7389 * L P A-0.3022^{*} L T P+42.6687 * L A W+0.0514 * L I A M L+0.2374 * L I E P A P+0.0878^{*}\right.$} \\
\hline & & LIEFI -311.0248 & 5*@TREND ) & & \\
\hline \multirow[t]{7}{*}{$L H S B$} & $L P A$ & 1.002297 & 1.196708 & 0.837545 & 0.4154 \\
\hline & $L T P$ & -1.029767 & 0.507785 & -2.027960 & 0.0607 \\
\hline & $L A W$ & 7.882963 & 7.168357 & 1.099689 & 0.2888 \\
\hline & LIAML & -0.395454 & 0.224133 & -1.764368 & 0.0980 \\
\hline & LIEPAP & 0.324330 & 0.171920 & 1.886518 & 0.0787 \\
\hline & $\mathrm{C}$ & -48.932703 & 47.665587 & -1.026583 & 0.3209 \\
\hline & \multicolumn{5}{|c|}{$-1.0298^{*} L T P+7.8830^{*} L A W-0.3955^{*}$ LIAML + 0.3243* LIEPAP-48.9327 ) } \\
\hline \multirow[t]{5}{*}{$L M t P$} & $L B C$ & 2.054836 & 3.147494 & 0.652848 & 0.5231 \\
\hline & $L S G$ & 1.103818 & 3.039592 & 0.363147 & 0.7212 \\
\hline & $L B$ & -0.439306 & 0.373729 & -1.175464 & 0.2570 \\
\hline & $\mathrm{C}$ & -17.376641 & 5.121563 & -3.392840 & 0.0037 \\
\hline & \multicolumn{5}{|c|}{ Cointeq $=L M t P-\left(2.0548^{*} L B C+1.1038^{*} L S G \quad-0.4393^{*} L B\right.$} \\
\hline \multirow[t]{3}{*}{$L M k P$} & $L B C$ & 1.631792 & 0.308266 & 5.293451 & 0.0000 \\
\hline & $\mathrm{C}$ & -4.851460 & 2.490795 & -1.947756 & 0.0656 \\
\hline & \multicolumn{5}{|c|}{ Cointeq $=L M k P-\left(1.6318^{*} L B C-4.8515\right)$} \\
\hline \multirow[t]{4}{*}{$L W P$} & $L B$ & 0.414660 & 0.260732 & 1.590370 & 0.1283 \\
\hline & $L P A$ & 1.211493 & 0.525871 & 2.303785 & 0.0327 \\
\hline & $\mathrm{C}$ & -4.433560 & 3.027189 & -1.464580 & 0.1594 \\
\hline & \multicolumn{5}{|c|}{ Cointeq $=L W P-\left(0.4147 * L B+1.2115^{*} L P A-4.4336\right)$} \\
\hline
\end{tabular}

The outcomes were explained in Table 5. 
4.4 ARDL Model. ARDL-Results Error Correction (Short Run) Model

Table 6. Coefficients ARDL model

\begin{tabular}{|c|c|c|c|c|c|c|c|c|c|c|c|}
\hline & Model 1 & Model 2 & Model 3 & Model 4 & Model 5 & Model 6 & Model 7 & Model 8 & Model 9 & Model 10 & Model 11 \\
\hline$D M P(-1)$ & -0.437344 & & & & & & & & & & \\
\hline$M P$ & 0.468559 & & & & & & & & & & \\
\hline $\operatorname{DPCL}(-1)$ & & -0.337866 & & & & & & & & & \\
\hline$P C L$ & & 0.504374 & & & & & & & & & \\
\hline$D L H A W(-1)$ & & & -0.549072 & & & & & & & & \\
\hline LHAW & & & 0.861523 & & & & & & & & \\
\hline$D L C P(-1)$ & & & & -0.357183 & & & & & & & \\
\hline$L C P$ & & & & 0.445034 & & & & & & & \\
\hline$D L H V(-1)$ & & & & & -0.163041 & & & & & & \\
\hline$L H V$ & & & & & 0.805676 & & & & & & \\
\hline DLHFB $(-1)$ & & & & & & -0.274946 & & & & & \\
\hline$L H F B$ & & & & & & 0.424065 & & & & & \\
\hline$D L H G(-1)$ & & & & & & & -0.659486 & & & & \\
\hline$L H G$ & & & & & & & -0.124458 & & & & \\
\hline$D L H S B(-1)$ & & & & & & & & -0.849175 & & & \\
\hline$L H S B$ & & & & & & & & 1.082191 & & & \\
\hline$D L M t P(-1)$ & & & & & & & & & -0.111604 & & \\
\hline$L M t P$ & & & & & & & & & 0.082670 & & \\
\hline$D L M k P(-1)$ & & & & & & & & & & 0.164587 & \\
\hline$L M k P$ & & & & & & & & & & -0.009118 & \\
\hline$D L W P(-1)$ & & & & & & & & & & & -0.043939 \\
\hline$L W P$ & & & & & & & & & & & 0.138804 \\
\hline$D L B C$ & & & & & & & & & 0.830547 & 0.109013 & \\
\hline$D L S G$ & & & & & & & & & 0.402297 & & \\
\hline$D L B$ & & & & & & & & & 0.068482 & & 0.196362 \\
\hline$L B C$ & 0.580681 & & & & & & & & -0.278581 & 0.000672 & \\
\hline$L S G$ & & & & & & & & & 0.265494 & & \\
\hline$L B$ & & & & & & & & & -0.059995 & & 0.026714 \\
\hline$D L P A$ & 0.594545 & 0.783195 & 0.044960 & 0.392275 & 0.415085 & & -0.750729 & 3.563394 & & & 0.272092 \\
\hline$D L T P$ & -0.066996 & -0.208843 & 0.027866 & -0.149153 & -0.131104 & & 0.451781 & -2.003352 & & & \\
\hline$A W$ & 2.581221 & -1.363666 & 0.803528 & 2.774211 & 2.714445 & & -1.316579 & 40.46012 & & & \\
\hline DLIAML & -0.054175 & 0.040287 & 0.019236 & 0.049452 & -0.017428 & 0.038012 & -0.044960 & -0.155800 & & & \\
\hline DLIEPAP & 0.114799 & & 0.011786 & 0.079863 & 0.082827 & 0.032582 & -0.108723 & 0.418113 & & & \\
\hline DLIEFI & -0.010697 & & 0.031669 & 0.078288 & 0.415085 & -0.063366 & -0.140212 & & & 0.002853 & \\
\hline$L P A$ & -0.815803 & -0.431030 & 0.383223 & -0.293663 & -0.131104 & & -0.156860 & 1.312192 & & & -0.230959 \\
\hline$L T P$ & 0.118491 & 0.228220 & 0.086748 & 0.133060 & 2.714445 & & -0.340704 & 1.674977 & & & \\
\hline$L A W$ & 1.170691 & 0.935868 & -1.889671 & 2.353774 & -0.017428 & & -2.898081 & -0.260007 & & & \\
\hline LIAML & 0.079012 & -0.044057 & 0.006904 & -0.072456 & 0.082827 & -0.049923 & 0.104635 & -0.180047 & & & \\
\hline LIEPAP & -0.227911 & & -0.013664 & -0.045954 & 0.415085 & -0.026900 & 0.005358 & -0.219591 & & & \\
\hline LIEFI & -0.012153 & & -0.091187 & -0.114110 & & $2.80 \mathrm{E}-05$ & 0.125274 & & & 0.000570 & \\
\hline$C$ & -5.257942 & -9.616749 & 5.692757 & -17.48425 & -4.582364 & -2.005777 & 24.58894 & -27.25057 & -0.229272 & 0.087282 & 0.580227 \\
\hline
\end{tabular}

The results of ARDL model coefficients (Table 6). 
Table 7. Estimates of the correlation coefficients (long-term and short-term)

\begin{tabular}{|c|c|c|c|c|c|c|c|c|c|c|c|}
\hline & Model 1 & Model 2 & Model 3 & Model 4 & Model 5 & Model 6 & Model 7 & Model 8 & Model 9 & Model 10 & Model 11 \\
\hline$D M P(-1)$ & 0.835256 & & & & & & & & & & \\
\hline $\operatorname{DPCL}(-1)$ & & -0.089364 & & & & & & & & & \\
\hline$D L H A W(-1)$ & & & 0.340866 & & & & & & & & \\
\hline$D L C P(-1)$ & & & & 0.309127 & & & & & & & \\
\hline$D L H V(-1)$ & & & & & -0.100540 & & & & & & \\
\hline $\operatorname{DLHFB}(-1)$ & & & & & & -0.289217 & & & & & \\
\hline$D L H G(-1)$ & & & & & & & 0.245767 & & & & \\
\hline DLHSB $8(-1)$ & & & & & & & & 0.254903 & & & \\
\hline$D L M t P(-1)$ & & & & & & & & & 0.028095 & & \\
\hline $\operatorname{DLMkP}(-1)$ & & & & & & & & & & 0.152961 & \\
\hline$D L W P(-1)$ & & & & & & & & & & & -0.032334 \\
\hline$D L B C$ & & & & & & & & & -0.080204 & & \\
\hline$D L S G$ & & & & & & & & & 0.349820 & & \\
\hline$D L B$ & & & & & & & & & 0.070588 & 0.024456 & 0.097499 \\
\hline$D L P A$ & -0.178966 & 0.937083 & 0.369308 & 0.087747 & 0.207382 & & -0.202667 & -0.311982 & -0.089801 & & 0.319188 \\
\hline$D L T P$ & -0.292370 & -0.214101 & 0.059442 & -0.174340 & -0.060702 & & 0.132118 & -0.270396 & & & \\
\hline$D L A W$ & 10.69479 & -2.502179 & -6.002750 & 0.820588 & 4.425060 & & 4.880925 & -3.742556 & -0.625368 & & \\
\hline DLIAML & 0.025156 & 0.034191 & 0.025874 & 0.006788 & -0.010195 & 0.028884 & -0.028801 & 0.034728 & & & \\
\hline DLIEPAP & 0.119206 & & -0.044324 & 0.102522 & 0.082063 & 0.017245 & -0.084309 & 0.081158 & -0.008268 & & \\
\hline DLIEFI & 0.084189 & & 0.043484 & 0.117222 & & -0.056691 & -0.141276 & & 0.008784 & 0.002541 & \\
\hline$E C T(-1)$ & -0.697214 & -0.496543 & -0.689227 & -0.620078 & -0.193119 & -0.524031 & -0.557041 & -0.931577 & -0.284903 & -0.006221 & -0.205384 \\
\hline C & -0.120952 & 0.037047 & 0.047544 & -0.012666 & -0.005897 & 0.049796 & 0.008864 & 0.050303 & 0.041405 & 0.032860 & 0.045709 \\
\hline
\end{tabular}

The resuls of short-term and ECM model have been illustrated (Table 7).

On the other hand, etc. coefficient is negative in all cases. Although ECM coefficient factors are not important, according to Pesaran and others (2001) they pave the way for having the cointegration relations because of negativity.

\subsection{Diagnostic Test}

Table 8. Diagnostic test results (F and LM Version)

\begin{tabular}{|c|c|c|c|c|c|c|c|c|}
\hline & \multicolumn{2}{|c|}{$\begin{array}{c}\text { Heteroskedasticity Test: } \\
\text { ARCH }\end{array}$} & \multicolumn{2}{|c|}{$\begin{array}{l}\text { Heteroskedasticity Test: } \\
\text { Breusch-Pagan-Godfrey }\end{array}$} & \multicolumn{2}{|c|}{$\begin{array}{l}\text { Breusch-Godfrey LM test } \\
\text { for serial correlation }\end{array}$} & \multirow[t]{2}{*}{$\begin{array}{l}\text { Jarque-Bera } \\
\text { Probability }\end{array}$} & \multirow{2}{*}{$\begin{array}{c}\text { GUSUM / } \\
\text { GUSUM of } \\
\text { Squares }\end{array}$} \\
\hline & F-Statistic & $\begin{array}{l}\text { Observed } \\
\text { R-Squared }\end{array}$ & F-Statistic & $\begin{array}{l}\text { Observed } \\
\text { R-Squared }\end{array}$ & F-Statistic & $\begin{array}{l}\text { Observed } \\
\text { R-Squared }\end{array}$ & & \\
\hline$L M P$ & $\begin{array}{c}0.689678 \\
(0.4161)\end{array}$ & $\begin{array}{r}0.733357 \\
(0.3918)\end{array}$ & $\begin{array}{c}0.542666 \\
(0.8467)\end{array}$ & $\begin{array}{l}10.10655 \\
(0.6852)\end{array}$ & $\begin{array}{c}0.420526 \\
(0.6723)\end{array}$ & $\begin{array}{c}2.467040 \\
(0.2913)\end{array}$ & $\begin{array}{c}4.373767 \\
(0.112266)\end{array}$ & $\begin{array}{l}\text { stability/ } \\
\text { stability }\end{array}$ \\
\hline$L P C L$ & $\begin{array}{c}0.402523 \\
(0.5330)\end{array}$ & $\begin{array}{c}0.434040 \\
(0.5100)\end{array}$ & $\begin{array}{c}0.605442 \\
(0.7429)\end{array}$ & $\begin{array}{c}5.066828 \\
(0.6518)\end{array}$ & $\begin{array}{c}1.202614 \\
(0.3317)\end{array}$ & $\begin{array}{c}3.591004 \\
(0.1660)\end{array}$ & $\begin{array}{c}1.347036 \\
(0.509912)\end{array}$ & $\begin{array}{l}\text { stability/ } \\
\text { stability }\end{array}$ \\
\hline LHAW & $\begin{array}{l}0.652621 \\
(0.4287)\end{array}$ & $\begin{array}{c}0.695198 \\
(0.4042)\end{array}$ & $\begin{array}{c}0.341436 \\
(0.9220)\end{array}$ & $\begin{array}{l}3.161071 \\
(0.8697)\end{array}$ & $\begin{array}{l}1.865424 \\
(0.1940)\end{array}$ & $\begin{array}{l}5.128820 \\
(0.0770)\end{array}$ & $\begin{array}{c}0.096543 \\
(0.952875)\end{array}$ & $\begin{array}{l}\text { stability/ } \\
\text { stability }\end{array}$ \\
\hline$L C P$ & $\begin{array}{c}0.562288 \\
(0.4621)\end{array}$ & $\begin{array}{c}0.601603 \\
(0.4380)\end{array}$ & $\begin{array}{l}0.753968 \\
(0.6466)\end{array}$ & $\begin{array}{l}6.925513 \\
(0.5447)\end{array}$ & $\begin{array}{c}0.901402 \\
(0.4318)\end{array}$ & $\begin{array}{l}3.004063 \\
(0.2227)\end{array}$ & $\begin{array}{c}3.474044 \\
(0.176044)\end{array}$ & $\begin{array}{l}\text { stability/ } \\
\text { stability }\end{array}$ \\
\hline$L H V$ & $\begin{array}{c}0.082566 \\
(0.7768)\end{array}$ & $\begin{array}{c}0.090449 \\
(0.7636)\end{array}$ & $\begin{array}{c}2.205338 \\
(0.1097)\end{array}$ & $\begin{array}{c}16.69242 \\
(0.1615)\end{array}$ & $\begin{array}{c}1.937024 \\
(0.2060)\end{array}$ & $\begin{array}{c}7.504020 \\
(0.0235)\end{array}$ & $\begin{array}{c}0.957749 \\
(0.619480)\end{array}$ & $\begin{array}{l}\text { stability/ } \\
\text { stability }\end{array}$ \\
\hline$L H F B$ & $\begin{array}{c}3.716349 \\
(0.0652)\end{array}$ & $\begin{array}{c}3.198018 \\
(0.0505)\end{array}$ & $\begin{array}{c}1.615326 \\
(0.2068)\end{array}$ & $\begin{array}{c}8.676449 \\
(0.1926)\end{array}$ & $\begin{array}{c}0.615658 \\
(0.5543)\end{array}$ & $\begin{array}{c}1.859343 \\
(0.3947)\end{array}$ & $\begin{array}{c}0.950244 \\
(0.621809)\end{array}$ & $\begin{array}{l}\text { stability/ } \\
\text { stability }\end{array}$ \\
\hline$L H G$ & $\begin{array}{c}3.010162 \\
(0.0981)\end{array}$ & $\begin{array}{c}2.878014 \\
(0.0898)\end{array}$ & $\begin{array}{c}1.842642 \\
(0.1705)\end{array}$ & $\begin{array}{c}15.83750 \\
(0.1988)\end{array}$ & $\begin{array}{c}8.947005 \\
(0.0091)\end{array}$ & $\begin{array}{c}15.89411 \\
(0.0004)\end{array}$ & $\begin{array}{c}1.483988 \\
(0.476164)\end{array}$ & $\begin{array}{l}\text { stability/ } \\
\text { stability }\end{array}$ \\
\hline$L H S B$ & 0.679472 & 0.722861 & 0.919705 & 6.907030 & 0.479179 & 1.579142 & 4.245980 & stability/ \\
\hline
\end{tabular}




\begin{tabular}{ccccccccc} 
& $(0.4195)$ & $(0.3952)$ & $(0.5184)$ & $(0.4386)$ & $(0.6298)$ & $(0.4540)$ & $(0.119673)$ & no stability \\
$L M t P$ & 1.128286 & 1.174837 & 1.119387 & 6.800193 & 3.060068 & 6.996132 & 0.857478 & stability/ \\
& $(0.3008)$ & $(0.2784)$ & $(0.3944)$ & $(0.3397)$ & $(0.0790)$ & $(0.0303)$ & $(0.651330)$ & no stability \\
$L$ & 0.962387 & 1.010024 & 0.017797 & 0.040861 & 0.264201 & 0.655926 & 1.373572 & stability/ \\
& $(0.3383)$ & $(0.3149)$ & $(0.9824)$ & $(0.9798)$ & $(0.7707)$ & $(0.7704)$ & $(0.503191)$ & no stability \\
\multirow{2}{*}{$L W P$} & 0.955526 & 1.003151 & 1.651524 & 4.757135 & 1.523206 & 3.495262 & 1.619058 & stability/ \\
& $(0.3400)$ & $(0.3165)$ & $(0.2111)$ & $(0.1905)$ & $(0.2463)$ & $(0.1742)$ & $(0.445068)$ & stability \\
\hline
\end{tabular}

Regression equations are adequate. It also passes all the diagnostic tests against serial correlation (Durbin Watson test and Breusch-Godfrey test), heteroscedasticity (White Heteroskedasticity Test), and normality of errors (Jarque-Bera test). The Ramsey RESET test also suggests that the model is well specified. All the results of these tests are shown in Table 8. The stability of the long-run coefficient is tested by the short-run dynamics. Once the ECM model has been estimated, the cumulative sum of recursive residuals (CUSUM) and the CUSUM of square (CUSUMSQ) tests are applied to assess the parameter stability (Pesaran and Pesaran, 1997). The results indicate the absence of any instability of the coefficients because the plot of the CUSUM and CUSUMSQ statistic fall inside the critical bands of the 5\% confidence interval of parameter stability.

\section{Conclusion}

The results of the study show that the gross agricultural output and gross agricultural output, the production of cereals and legumes, winter wheat, potatoes, vegetables, fruits and berries, grapes, sugar beets, meat, milk and eggs The number of people working in agriculture, forestry and fisheries, the number of cattle, sheep and goats, the number of agricultural machinery, as well as the number of agricultural machinery for land cultivation, agriculture, forestry and fisheries investment Intensive factors, such as the fleet of basic agricultural machinery, the processing of agricultural products and the import of equipment for the food industry, also need to be addressed.

\section{References}

Abdulkarim, A. K., Al-shihri, F. S., \& Ahmed, S. M. (2014). Inter-Sectoral Linkages and Economic Growth in Saudi Arabia: Toward a Successful Long-term Development Strategy. International Journal of Science and Research 3(8), 1654-1659. Retrieved from https://www.ijsr.net/archive/v3i8/MDIwMTU3ODk=.pdf

Alam, G. (2008). The role of technical and vocational education in the national development of Bangladesh. International Journal of Work-Integrated Learning, 9(1), 25. Retrieved from https://www.ijwil.org/files/APJCE_09_1_25_44.pdf

Anthony, E. (2010). Agricultural credit and economic growth in Nigeria: An empirical analysis. Business and Economics Journal, 14(1), 1-7.

Barnard, G. A. (1959). Control charts and stochastic processes. Journal of the Royal Statistical Society: Series B (Methodological), 21(2), 239-257. https://doi.org/10.1111/j.2517-6161.1959.tb00336.x

Breusch, T. S., \& Pagan, A. R. (1979). A simple test for heteroscedasticity and random coefficient variation. Econometrica: Journal of the Econometric Society, 1287-1294. https://doi.org/10.2307/1911963

Datt, G., \& Ravallion, M. (1998). Farm productivity and rural poverty in India. The Journal of Development Studies, 34(4), 62-85. https://doi.org/10.1080/00220389808422529

Dickey, D. A., \& Fuller, W. A. (1979). Distribution of the estimators for autoregressive time series with a unit root. Journal of the American Statistical Association, 74(366a), 427-431. https://doi.org/10.1080/01621459.1979.10482531

Dowrick, S., \& Gemmell, N. (1991). Industrialisation, catching up and economic growth: a comparative study across the world's capitalist economies. The Economic Journal, 101(405), 263-275. https://doi.org/10.2307/2233817

Eicher, C. K. (1990). Agricultural development in the Third World, edited by Carl K. Eicher \& John M. Staatz. The Johns Hopkins studies in development. Retrieved from https://ru.booksc.xyz/book/50056655/d86841

Gardner, B. L., Rausser, G. C., Pingali, P. L., \& Evenson, R. E. (Eds.) (2001). Handbook of Agricultural Economics: Agriculture and Its External Linkages (Vol. 2). Elsevier.

Gollin, D., Parente, S. L., \& Rogerson, R. (2002). The role of agriculture in development. American Economic Review, 
92(2), 160-164. https://doi.org/10.1257/000282802320189177

Im, K. S., Pesaran, M. H., \& Shin, Y. (2003). Testing for unit roots in heterogeneous panels. Journal of Econometrics, 115(1), 53-77.

Kwiatkowski, D., Phillips, P. C., Schmidt, P., \& Shin, Y. (1992). Testing the null hypothesis of stationarity against the alternative of a unit root root: How sure are we that economic time series have a unit root?. Journal of Econometrics, 54(1-3), 159-178. https://doi.org/10.1016/0304-4076(92)90104-Y

Levin, A., Lin, C. F., \& Chu, C. S. J. (2002). Unit root tests in panel data: asymptotic and finite-sample properties. Journal of Econometrics, 108(1), 1-24. https://doi.org/10.1016/S0304-4076(01)00098-7

MacKinnon, J. G. (1996). Numerical distribution functions for unit root and cointegration tests. Journal of Applied Econometrics, $11(6)$, 601-618. https://doi.org/10.1002/(SICI)1099-1255(199611)11:6<601::AID-JAE417>3.0.CO;2-T

Mirza Md. Moyen Uddin. (2015). Causal relationship between agriculture, industry and services sector for GDP growth in Bangladesh: An econometric investigation. Journal of Poverty, Investment and Development, 8, 124-130. Retrieved from http://citeseerx.ist.psu.edu/viewdoc/download?doi=10.1.1.676.6370\&rep=rep1\&type=pdf

Narayan, P. K. (2005). The saving and investment nexus for China: evidence from cointegration tests. Applied Economics, 37(17), 1979-1990. https://doi.org/10.1080/00036840500278103

Page, E. S. (1954). Continuous inspection schemes. Biometrika, 41(1/2), 100-115. https://doi.org/10.1093/biomet/41.1-2.100

Pesaran, M. H., \& Shin, Y. (1999). An Autoregressive Distributed Lag Modelling Approach to Cointegration Analysis. In S. Strom (Ed.), Econometrics and Economic Theory in the 20th Century: The Ragnar Frisch Centennial Symposium (chp. 11). Cambridge: Cambridge University Press. Retrieved from http://citeseerx.ist.psu.edu/viewdoc/download?doi=10.1.1.153.3246\&rep=rep1\&type=pdf

Pesaran, M. H., Shin, Y., \& Smith, R. J. (2001). Bounds testing approaches to the analysis of level relationships. Journal of Applied Econometrics, 16(3), 289-326. https://doi.org/10.1002/jae.616

Phillips, P. C., \& Perron, P. (1988). Testing for a unit root in time series regression. Biometrika, 75(2), 335-346. https://doi.org/10.1093/biomet/75.2.335

Schultz, T. W. (1964). Transforming traditional agriculture. Transforming traditional agriculture. Retrieved from https://www.cabdirect.org/cabdirect/abstract/19641802933

Schwarz, G. (1978). Estimating the dimension of a model. The Annals of Statistics, 6(2), 461-464. https://doi.org/10.1214/aos/1176344136

Singariya, M., \& Sinha, N. (2015). Relationships among per capita GDP, agriculture and manufacturing sectors in India. Journal of Finance and Economics, 3(2), 36-43.

Subramaniam, V., \& Reed, M. R. (2009). Agricultural inter-sectoral linkages and its contribution to economic growth in the transition countries (No. 1005-2016-79162). Contributed Paper Prepared for Presentation at the International Association of Agricultural Economists Conference, Beijing, China, August 16-22.

Thirtle, C., Lin, L., \& Piesse, J. (2003). The impact of research-led agricultural productivity growth on poverty reduction in Africa, Asia and Latin America. World Development, 31(12), 1959-1985. https://doi.org/10.1016/j.worlddev.2003.07.001

Timmer, C. P. (1995). Getting agriculture moving: do markets provide the right signals?. Food policy, 20(5), 455-482. https://doi.org/10.1016/0306-9192(95)00038-G

Timmer, C. P. (2002). Agriculture and economic development. Handbook of Agricultural Economics, 2, 1487-1546. https://doi.org/10.1016/S1574-0072(02)10011-9

Titus, O. A. (2009). Does agriculture really matter for economic growth in developing countries? (No. 319-20169808). Selected Paper prepared for presentation at the American Agricultural Economics Association Annual Meeting, $\quad$ Milwaukee, WI, July $28 . \quad$ Retrieved from http://citeseerx.ist.psu.edu/viewdoc/download?doi=10.1.1.457.5583\&rep=rep1\&type=pdf 
Tolulope O., \& Etumnu, C. (2013). Contribution of Agriculture to Economic Growth in Nigeria, The 18th Annual Conference of the African Econometric Society (AES) Accra, Ghana at the session organized by the Association for the Advancement of African Women Economists (AAAWE), 22nd and 23rd July. Retrieved from https://pdfs.semanticscholar.org/1b7b/284b899d9cc59eba44c29fbc6db898946320.pdf

Wilfrid, A. B. H. L., \& Edwige, K. (2004). Role of agriculture in economic development of developing countries: case study of China and Sub-Saharan Africa (SSA). Journal of Agriculture and Social Research (JASR), 4(2), 1-18. https://doi.org/10.4314/jasr.v4i2.2811

\section{Notes}

Note 1. This is an example.

Note 2. This is an example for note 2.

\section{Copyrights}

Copyright for this article is retained by the author(s), with first publication rights granted to the journal.

This is an open-access article distributed under the terms and conditions of the Creative Commons Attribution license (http://creativecommons.org/licenses/by/4.0/). 